\title{
VARIATION OF THE CANONICAL HEIGHT FOR POLYNOMIALS IN SEVERAL VARIABLES
}

\author{
PATRICK INGRAM
}

\begin{abstract}
Let $K$ be a number field, let $X / K$ be a curve, let $f_{t}: \mathbb{P}^{N} \rightarrow \mathbb{P}^{N}$ be a family of morphisms over $X$, and let $P: X \rightarrow \mathbb{P}^{N}$ be a morphism. Writing $\hat{h}_{f_{t}}$ for the canonical height associated to $f_{t}$, it follows from a result of Call and Silverman that $\hat{h}_{f_{t}}\left(P_{t}\right) / h_{X}(t)=\hat{h}_{f_{\eta}}\left(P_{\eta}\right)+o(1)$, where $\eta$ is the generic point of the curve. Assuming that there is a hyperplane $H$ such that $f_{\eta}^{*} H=d H$, and such that the restriction of the family to $H$ is isotrivial, we improve this estimate to

$$
\hat{h}_{f_{t}}\left(P_{t}\right)=\hat{h}_{f_{\eta}}\left(P_{\eta}\right) h_{X}(t)+O(1)
$$
\end{abstract}

for a particular Weil height $h$ on $X$. In the one-variable case, this reduces to an earlier result of the author.

Let $f: \mathbb{P}^{N} \rightarrow \mathbb{P}^{N}$ be a morphism of degree $d \geq 2$, defined over a number field $K$. There is, associated to $f$, a canonical height function $\hat{h}_{f}: \mathbb{P}^{N}(\bar{K}) \rightarrow \mathbb{R}$ which is roughly analogous to the Néron-Tate height on an abelian variety, and reveals much about the arithmetic dynamics of $f$. It is natural to ask how this function varies as one varies the morphism to which it is associated.

Call and Silverman [3] have shown that if $t \mapsto f_{t}$ is an algebraic family of endomorphisms of $\mathbb{P}^{N}$, over a base curve $X$, and $t \mapsto P_{t}$ is a family of points, then

$$
\hat{h}_{f_{t}}\left(P_{t}\right)=\left(\hat{h}_{f_{\eta}}\left(P_{\eta}\right)+o(1)\right) h_{X, D}(t)
$$

where $\eta \in X$ is the generic point, $D \in \operatorname{Div}(X)$ is any divisor of degree 1 , and $o(1) \rightarrow 0$ as $h_{X, D}(t) \rightarrow \infty$. The author [6] showed that the estimate (11) could be improved to

$$
\hat{h}_{f_{t}}\left(P_{t}\right)=\hat{h}_{f_{\eta}}\left(P_{\eta}\right) h_{X, D}(t)+O(1),
$$

for a specific divisor $D=D(f, P) \in \operatorname{Div}(X) \otimes \mathbb{Q}$ of degree 1 , but only for polynomial endomorphisms of $\mathbb{P}^{1}$ (remarkably, Ghioca and Mavraki 4 have recently extended this to certain non-polynomial rational functions). The estimate (2) is similar to a result of Tate 8 in the context of elliptic surfaces, and is in some sense best possible, since Weil heights are generally viewed as $O(1)$-equivalence classes. This note provides a generalization of the main result of [6] to the case of polynomial maps in several variables. Note that for any morphism $f: \mathbb{P}^{N} \rightarrow \mathbb{P}^{N}$ and any hyperplane $H \subseteq \mathbb{P}^{N}$, we have $f^{*} H \sim d H$ for some integer $d \geq 1$. We will say that $f$ is a regular polynomial endomorphism with respect to $H$ if this linear equivalence turns out to be an equality. For a study of the complex dynamics of regular polynomial endomorphisms of $\mathbb{P}^{N}$, see Bedford and Jonsson [2].

There is a natural map from the space of regular polynomial endomorphisms of $\mathbb{P}^{N}$ to the moduli space of endomorphisms of $\mathbb{P}^{N-1}$ of the same degree, obtained

Date: January 16, 2021. 
by restricting to the invariant hyperplane, and we will say that a family $f_{t}$ over $X$ is fibral if and only if its image is contained in a single fibre of this projection.

Theorem 1. Let $K$ be a number field, let $X / K$ be a projective curve, let $f_{t}: \mathbb{P}^{N} \rightarrow$ $\mathbb{P}^{N}$ be a fibral family of regular polynomial endomorphisms relative to $H$ over $X$ defined over $K$, and let $P: X \rightarrow \mathbb{P}^{N}$. There is a divisor $D=D(f, P) \in \operatorname{Div}(X) \otimes \mathbb{Q}$ of degree 1 such that

$$
\hat{h}_{f_{t}}\left(P_{t}\right)=\hat{h}_{f_{\eta}}\left(P_{\eta}\right) h_{X, D}(t)+O(1) .
$$

Although Theorem 1 applies to a specific divisor class, from it one can deduce a general result which improves (10) in the relevant cases. Note that the result is easily extended to divisors of arbitrary degree, by the linearity of the Weil height machine.

Corollary 2. Let $K, X, f$, and $P$ be as in Theorem 1. Then for any divisor $D \in \operatorname{Div}(X) \otimes \mathbb{Q}$ we have

$$
\operatorname{deg}(D) \hat{h}_{f_{t}}\left(P_{t}\right)=\hat{h}_{f_{\eta}}\left(P_{\eta}\right) h_{X, D}(t)+O\left(h_{X, D}(t)^{1 / 2}\right),
$$

where the error term may be further improved to $O(1)$ if $X$ is rational.

We note that in the case $N=1$, the hypothesis that family be fibral automatically obtains, since $H$ is a point. In the case $N \geq 2$, this assumption is non-trivial, and is used to ensure sufficient regularity of local heights. Specifically, the fact that all polynomials in one variable behave similarly close to the totally invariant point grants a great deal of uniformity in the construction of dynamical Green's functions. In dimension $N \geq 2$, this is no longer true, but when we restrict attention to families for which it is, a similar regularity of Green's functions emerges. We note that the condition may be presented in a more elementary, but coordinate dependent, fashion. If $f \in K(X)\left(\mathbb{P}^{N}\right)$ is written in coordinates as

$$
f_{i}\left(x_{0}, \ldots, x_{N}\right)=\sum_{j_{0}+\cdots+j_{N}=d} c_{i,\left(j_{0}, \ldots, j_{N}\right)} x_{0}^{j_{0}} \cdots x_{N}^{j_{N}}
$$

for $0 \leq i \leq N-1$, with $c_{i,\left(j_{0}, \ldots, j_{N}\right)} \in K(X)$ and $f_{N}=x_{N}^{d}$, then $f$ is polynomial with respect to the hyperplane $H$ defined by $x_{N}=0$. The family is then fibral if (but not only if) $c_{i,\left(j_{0}, \ldots, j_{N}\right)}$ for all indices with $j_{N}=0$.

Remark 1 . The condition of fibrality of the family in Theorem 1 seems initially to be quite strong, but we note that eliminating it implies something much stronger. In particular, suppose that Theorem 1 holds for arbitrary families of regular polynomial endomorphisms, and let $f / X: \mathbb{P}^{N} \rightarrow \mathbb{P}^{N}$ be any family of endomorphisms (regular or otherwise). It is easy to see that $f$ is the restriction to the invariant hyperplane of a family $\tilde{f}$ of regular polynomial endomorphisms of $\mathbb{P}^{N+1}$. If $f=\left[f_{0}: \cdots: f_{N}\right]$, we may simply set $\tilde{f}=\left[f_{0}: \cdots f_{N}: x_{N+1}^{\operatorname{deg}(f)}\right]$. It is also easy to check that, for any $P \in \mathbb{P}^{N}(X)$ we have $\hat{h}_{f_{t}}\left(P_{t}\right)=\hat{h}_{\tilde{f}_{t}}\left(\tilde{P}_{t}\right)$, where $\tilde{P}$ is the image of $P$ under the obvious isomorphism $\mathbb{P}^{N} \cong\left\{x_{N+1}=0\right\} \subseteq \mathbb{P}^{N+1}$. In other words, if Theorem 1 can be extended to arbitrary families if regular polynomial endomorphisms of $\mathbb{P}^{N}$, for any $N$, then it can also be extended to arbitrary families of morphisms. 
It follows immediately from Theorem 1 that if $f$ and $P$ are as in the theorem, and $\hat{h}_{f_{\eta}}\left(P_{\eta}\right)>0$, then the set

$$
\left\{t \in X(\bar{K}): P_{t} \text { is preperiodic for } f_{t}\right\}
$$

is a set of bounded height, and hence contains only finitely many points of any given algebraic degree over $K$, but in many cases something stronger is true. For instance, Baker and DeMarco [1] have shown that if

$$
f_{t}(x, y, z)=\left[x^{d}+t z^{d}: y^{d}+t z^{d}: z^{d}\right],
$$

and $P_{t}=(a, b)$ for fixed $a, b \in K$, then the set defined above is actually finite unless $a^{d}=b^{d}$. One might speculate, then, that the set defined in (3) is finite in general when $N \geq 2$, unless the dynamics decomposes into lower-dimensional parts which are dependent in some strong sense.

In Section 1 we construct dynamical Green's function associated to polynomial morphisms $\mathbb{P}^{N} \rightarrow \mathbb{P}^{N}$ and show that these local heights are fairly uniform for polynomials which are "the same at infinity." In Section 3 we study the variation of these heights in families, giving a proof of Theorem 1 .

\section{Green's Functions}

Let $K$ be a field, and let $M_{K}$ be a set of valuations on $K$. For each $v \in M_{K}$, we denote by $\mathbb{C}_{v}$ the smallest extension of $K$ which is both algebraically closed, and complete with respect to $v$. By an $M_{K}$-constant $\gamma$ we mean a function $\gamma: M_{K} \rightarrow \mathbb{R}$ such that $\gamma_{v}=0$ for all but a finite number of $v \in M_{K}$. A collection of functions $\varphi_{v}: X\left(\mathbb{C}_{v}\right) \rightarrow \mathbb{R}$ will be called $M_{K}$-bounded if there is an $M_{K^{-}}$-constant $\gamma$ such that $\left|\varphi_{v}(t)\right| \leq \gamma_{v}$ for all $t \in X\left(\mathbb{C}_{v}\right)$, for all $v \in M_{K}$; we will often denote $M_{K^{-}}$ boundedness by $\varphi_{v}(t)=O_{v}(1)$, but observe that the $O_{v}(1)$ notation here implies both that the constant bound depends on $v$, and that it vanishes for almost all $v$. For convenience we note that if $\gamma_{1}, \gamma_{2}$ are $M_{K}$-constants then so are $\max \left\{\gamma_{1}, \gamma_{2}\right\}$, $\min \left\{\gamma_{1}, \gamma_{2}\right\}$, and $\gamma_{1}+c \gamma_{2}$, for any $c \in \mathbb{R}$.

Let $f: \mathbb{P}^{N} \rightarrow \mathbb{P}^{N}$ be a morphism satisfying $f^{*} H=d H$, for the hyperplane $H=\left\{x_{N}=0\right\}$. For a point in homogeneous coordinates

$$
P=\left[x_{0}: \cdots: x_{N}\right] \in \mathbb{A}^{N}=\mathbb{P}^{N} \backslash H,
$$

we define

$$
\|P\|_{v}=\frac{\max \left\{\left|x_{i}\right|_{v}: 0 \leq i \leq N-1\right\}}{\left|x_{N}\right|_{v}},
$$

which is independent of homogeneous scaling.

By a multi-index $I$ of dimension $N$ we mean a tuple $\left(i_{0}, \ldots, i_{N}\right)$, and we write $|I|=i_{0}+\cdots+i_{N}$. If $\mathbf{x}=\left(x_{0}, \ldots, x_{N}\right)$ is a tuple of variables, and $I=\left(i_{0}, \ldots, i_{N}\right)$ is a multi-index, then $\mathbf{x}^{I}$ is an abbreviation for the monomial $x_{0}^{i_{0}} \cdots x_{N}^{i_{N}}$. Given that $f^{*} H=d H$, we may assume without loss of generality that $f_{N}(\mathbf{x})=x_{N}^{d}$, and write the other components of $f$ in coordinates as

$$
f_{i}(\mathbf{x})=\sum_{|I|=d} a_{i, I} \mathbf{x}^{I} .
$$

Once this representation is fixed, we set

$$
\mathcal{B}_{v}(f)=\log ^{+} \max \left\{\left|a_{i, I}\right|^{1 / I_{N}}: 0 \leq i \leq N-1, I_{N} \neq 0\right\} .
$$


We note, to aid the reader's intuition, that if $f$ is a monic polynomial in one variable, and $v$ is non-archimedean, then $\mathcal{B}_{v}(f)=\log ^{+} \max \left\{|\alpha|_{v}: f(\alpha)=0\right\}$.

Following the standard convention, we will define a Green's function $G_{f}: \mathbb{A}_{\mathbb{C}_{v}}^{N} \rightarrow$ $\mathbb{R}$ by

$$
G_{f, v}(P)=\lim _{n \rightarrow \infty} d^{-n} \log ^{+}\left\|f^{n}(P)\right\|_{v}
$$

It is easy to show that both of these limits always exist, and we present a few other properties of these functions.

Lemma 3. Let $f: \mathbb{P}^{N} \rightarrow \mathbb{P}^{N}$ be a regular polynomial endomorphism defined over $K$. There exists an $M_{K}$-constant $\gamma$ such that if

$$
\log \|P\|_{v}>\mathcal{B}_{v}(f)+\gamma_{v},
$$

then

$$
G_{f, v}(P)=\log \|P\|_{v}+O_{v}(1) .
$$

Furthermore, both $\gamma$ and the implied $M_{K}$-constant above depend only on the restriction of $f$ to the invariant hyperplane.

Proof. Write the coordinate functions of $f$ as $f_{i}=\sum_{|I|=d} a_{i, I} \mathbf{x}^{I} \in K[\mathbf{x}]$, as above, and let $g_{i}(\mathbf{x})=\sum_{I_{N}=0} a_{i, I} \mathbf{x}^{I}$. In other words, $g_{i}(\mathbf{x})=f_{i}\left(x_{0}, \ldots, x_{N-1}, 0\right)$, the highest-degree homogeneous summand of $f_{i}\left(x_{0}, \ldots, x_{N-1}, 1\right)$, and quantities which depend only on the $g_{i}$ depend only on the restriction of $f$ to $H$.

We will choose our $M_{K}$ constant $\gamma$ by insisting first that $\gamma_{v} \geq 0$ for all $v$, and then increasing the value of $\gamma_{v}$ as necessary. Let $P_{0}, \ldots, P_{N} \in \mathbb{C}_{v}$ with $P_{N} \neq 0$, let $P=\left[P_{0}: \cdots: P_{N}\right]$, and for a multi-index $I$ write $P^{I}$ for $\prod P_{k}^{I_{k}}$.

If we have $\log \|P\|_{v}>\mathcal{B}_{v}(f)+\gamma_{v}$, then for any $i$ and any $I$ with $I_{N} \neq 0$, we have

$$
\log \left|a_{i, I}\right|_{v} \leq \log \left|a_{i, I}\right|_{v}+\gamma_{v} \leq \log \left|a_{i, I}\right|_{v}+I_{N} \gamma_{v}<I_{N} \log \|P\|_{v} .
$$

It follows from this that

$$
\begin{aligned}
\log \left|a_{i, I} P^{I}\right|_{v} & \leq \log \left|a_{i, I}\right|_{v}+\left(d-I_{N}\right) \log \max \left\{\left|P_{0}\right|_{v}, \ldots,\left|P_{N-1}\right|_{v}\right\}+I_{N} \log \left|P_{N}\right|_{v} \\
& \leq d \log \left|P_{N}\right|_{v}+\left(d-I_{N}\right) \log \|P\|_{v}+\log \left|a_{i, I}\right|_{v} \\
& <d \log \|P\|_{v}+d \log \left|P_{N}\right|_{v}-\gamma_{v} .
\end{aligned}
$$

Now let $C_{1, v}=\log \left(\begin{array}{c}n+d-1 \\ d-1\end{array}\right)$ if $v$ is archimedean, and $C_{1, v}=0$ otherwise. For each $i$, still subject to $\log \|P\|_{v}>\mathcal{B}_{v}(f)+\gamma_{v}$, we have

$$
\begin{aligned}
\log \left|f_{i}(P)-g_{i}(P)\right|_{v} & =\log \left|\sum_{I_{N} \neq 0} a_{i, I} P^{I}\right|_{v} \\
& \leq \log \max _{I_{N} \neq 0}\left|a_{i, I} P^{I}\right|_{v}+C_{1, v} \\
& <d \log \|P\|_{v}+d \log \left|P_{N}\right|_{v}-\gamma_{v}+C_{1, v} .
\end{aligned}
$$

By a standard argument from the Nullstellensatz [5, ?], there exists an $M_{K^{-}}$ constant $C_{2}$ (depending only on the $g_{j}$ ) such that

$$
\left|d \log \max \left\{\left|P_{0}\right|_{v}, \ldots,\left|P_{N-1}\right|_{v}\right\}-\log \max \left\{\left|g_{0}(P)\right|_{v}, \ldots,\left|g_{N-1}(P)\right|_{v}\right\}\right| \leq C_{2, v} .
$$

It follows that, for some $0 \leq i \leq N-1$,

$$
\log \left|g_{i}(P)\right|_{v} \geq d \log \|P\|_{v}+d \log \left|P_{N}\right|-C_{2, v} .
$$


Now let $C_{3, v}=\log 2$ if $v$ is archimedean, and $C_{3, v}=0$ otherwise. Combining (4) with (6), we see that for the index $i$ in (6) we have

$$
\log \left|g_{i}(P)\right|_{v}-\log \left|f_{i}(P)-g_{i}(P)\right|_{v}-C_{3, v}>\gamma_{v}-C_{1, v}-C_{2, v}-C_{3, v},
$$

and we increase $\gamma$ to ensure that the right-hand-side is never negative. This now implies $\left|g_{i}(P)\right|_{v}>e^{C_{3, v}}\left|f_{i}(P)-g_{i}(P)\right|_{v}$, which by the triangle inequality gives

$$
\left|f_{i}(P)\right|_{v} \geq \frac{1}{e^{C_{3, v}}}\left|g_{i}(P)\right|_{v}
$$

It follows that

$$
\begin{aligned}
\log \|f(P)\|_{v} & \geq \log \left|g_{i}(P)\right|_{v}-C_{3, v}-\log \left|P_{N}^{d}\right|_{v} \\
& \geq d \log \|P\|_{v}-C_{2, v}-C_{3, v} .
\end{aligned}
$$

At the same time, applying (5) in the other direction gives

$$
\begin{aligned}
\log \|f(P)\|_{v} & =\max \left\{\left|f_{j}(P)\right|_{v}\right\}-d \log \left|P_{N}\right|_{v} \\
& \leq \max \left\{\log \left|f_{j}(P)-g_{j}(P)\right|_{v}, \log \left|g_{j}(P)\right|_{v}\right\}+C_{3, v}-d \log \left|P_{N}\right|_{v} \\
& \leq d \log \|P\|_{v}+C_{3, v}+\max \left\{C_{1, v}-\gamma_{v}, C_{2, v}\right\} .
\end{aligned}
$$

Now set

$$
C_{4, v}=\max \left\{C_{3, v}+\max \left\{C_{1, v}-\gamma_{v}, C_{2, v}\right\}, C_{2, v}+C_{3, v}\right\} .
$$

Note that $C_{4}$ is an $M_{K}$-constant, and since $C_{1}, C_{2}$, and $C_{3}$ are independent of $\gamma$, increasing the value of $\gamma$ does not increase the value of $C_{4}$. (Indeed, since $\gamma \geq 0$ by our initial choice, we might just as easily chosen something like $C_{4}=$ $C_{3}+\max \left\{C_{1}, C_{2}, 0\right\}$.)

We thus have an $M_{K}$-constant $C_{4}$ depending only on the restriction of $f$ to $H$ such that for all $v$,

$$
-C_{4, v} \leq \log \|f(P)\|_{v}-d \log \|P\|_{v} \leq C_{4, v}
$$

whenever $\log \|P\|_{v}>\mathcal{B}_{v}(f)+\gamma_{v}$. If we suppose that $\gamma \geq C_{4} /(d-1)$, then this means $\log \|P\|_{v}>\mathcal{B}_{v}(f)+\gamma_{v}$ also implies

$$
\log \|f(P)\|_{v} \geq d \log \|P\|_{v}-C_{4, v}>d \mathcal{B}_{v}(f)+d \gamma_{v}-C_{4, v}>\mathcal{B}_{v}+(f) \gamma_{v},
$$

and so we may apply iteration to the estimate (7). A standard telescoping sum argument gives

$$
\begin{aligned}
\left|G_{f, v}(P)-\log \|P\|_{v}\right| & \leq \sum_{n=0}^{\infty}\left|d^{-(n+1)} \log \left\|f^{n+1}(P)\right\|_{v}-d^{-n} \log \left\|f^{n}(P)\right\|_{v}\right| \\
& \leq \frac{C_{4, v}}{d}+\frac{C_{4, v}}{d^{2}}+\frac{C_{4, v}}{d^{3}}+\cdots \\
& =\frac{1}{d-1} C_{4, v} .
\end{aligned}
$$

This proves the lemma.

If $K$ is a global field and $M_{K}$ is its set of places, then as usual 3 we define the canonical height on $\mathbb{P}^{N}$ associated to $f$ by

$$
\hat{h}_{f}(P)=\lim _{n \rightarrow \infty} d^{-n} h\left(f^{n}(P)\right) .
$$


It is easy to show that, for any finite extension $L / K$ and any $P \in \mathbb{A}^{N}(L)$, we have

$$
\hat{h}_{f}(P)=\sum_{v \in M_{L}} \frac{\left[L_{v}: K_{v}\right]}{[L: K]} G_{f, v}(P)
$$

Specifically, if we have, for every place $v$ of $L$, that $\log ^{+}\|P\|_{v}=0$ or $\log \|P\|_{v}>$ $\mathcal{B}_{v}(f)+\gamma_{v}$, then by Lemma 3 we have that

$$
h_{\mathbb{P}^{N}, H}(P)=\sum_{v \in M_{L}} \frac{\left[L_{v}: K_{v}\right]}{[L: K]} \log \|P\|_{v}=\sum_{v \in M_{L}} \frac{\left[L_{v}: K_{v}\right]}{[L: K]} G_{f, v}(P)+O(1),
$$

where the implied constant depends on $f$ but not on $P$. But the antecedent condition is preserved by the application of $f$, and hence

$$
d^{-n} h\left(f^{n}(P)\right)=\sum_{v \in M_{L}} \frac{\left[L_{v}: K_{v}\right]}{[L: K]} G_{f, v}(P)+O\left(d^{-n}\right),
$$

where the implied constant is independent of $n$. Taking $n \rightarrow \infty$ eliminates the constant. The assumption that $\log ^{+}\|P\|_{v}=0$ or $\log \|P\|_{v}>\mathcal{B}_{v}(f)+\gamma_{v}$ for each $v$ can now be removed, since it holds for $f^{m}(P)$ when $m$ is sufficiently large.

\section{LOCAL HEIGHTS}

With local results now in hand, we establish the technical machinery needed for the proof of the main result. Throughout we work over two global fields, namely $K$, a number field, and the function field of a curve $X / K$. We will write $M_{K}$ for the set of places of $K$, and we will assume that for each $v \in K$ we have chosen a complete, algebraically closed extension $\mathbb{C}_{v}$. We will let $F=\bar{K}(X)$ be the function field of $X$ over $\bar{K} \subseteq \mathbb{C}_{v}$. Note that, if we take $X$ to be a smooth complete model of itself, the places $M_{F}$ of $F$ correspond exactly to points $\beta \in X(\bar{K})$. We normalize the absolute value associated to $\beta$ such that for any $\varphi \in F,-\log |\varphi|_{\beta}$ is the order to which $\varphi$ vanishes at $\beta$.

We recall from 7 the definition of a local height with respect to a Cartier divisor $D \in \operatorname{Div}(X)$, which is extended to $\operatorname{Div}(X) \otimes \mathbb{Q}$ by linearity. A Cartier divisor is represented by a collection of pairs $\left(U_{i}, g_{i}\right)$, where the $U_{i}$ offer a finite cover of $X$ by affine open sets, and the $g_{i}$ are rational functions on the $U_{i}$ subject to the condition that $g_{i} / g_{j}$ and $g_{j} / g_{i}$ are both regular on $U_{i} \cap U_{j}$. This allows us to define, unambiguously, $\operatorname{ord}_{\beta}(D)=-\log \left|g_{i}\right|_{\beta}$ for any $i$ with $\beta \in U_{i}$, and the Weil divisor described by this data is $\sum_{\beta \in X} \operatorname{ord}_{\beta}(D)[\beta]$.

A Néron divisor adds a collection of functions $\alpha_{i, v}: U_{i}\left(\mathbb{C}_{v}\right) \rightarrow \mathbb{R}$ which are continuous and locally $M_{K}$-bounded, and the local height associated to this data is given by

$$
\lambda_{X, D, v}(t)=-\log \left|g_{i}(t)\right|_{v}+\alpha_{i, v}(t) .
$$

The compatibility conditions are set out so that this is well-defined. Although the local height function depends on the Néron data associated to the Cartier divisor, one can show [7, Chapter 10] both that every Cartier divisor admits such a structure, and that changing the Néron divisor associated to a given Cartier divisor will perturb the local height only by an $M_{K}$-bounded amount.

The main theorem of this paper is almost an immediate consequence of the following local result, although in the next section we will see a few technical details emerge. Before stating the theorem, we explain the notation somewhat. Since $M_{F}$ corresponds to the set of $\bar{K}$-points on $X$, we have for each $\beta \in X(\bar{K})$ a value 
$G_{f_{\eta}, \beta}\left(P_{e} t a\right)$, where we simplify notation by confusing the point and the corresponding valuation.

Theorem 4. Let $K, X, f, P$ be as above,, let

$$
D=\sum_{\beta \in X} G_{f_{\eta}, \beta}\left(P_{\eta}\right)[\beta] \in \operatorname{Div}(X) \otimes \mathbb{Q},
$$

let $U \subseteq X$ an affine open set on which the coordinates of $P$ and the coefficients of $f$ are regular. Then we have

$$
G_{f_{t}, v}\left(P_{t}\right)=\lambda_{X, D, v}(t)+O_{v}(1)
$$

for all $v \in M_{K}$ and all $t \in U\left(\mathbb{C}_{v}\right)$ (where the implied constant vanishes for all but finitely many $v)$.

We restrict to an affine open subscheme of $X$ since one or both of the sides of (9) might be undefined at points where some coordinate of $P$ or some coefficient of $f$ is not regular. Also, it is worth noting that it is not a priori obvious that $G_{f_{\eta}, \beta}\left(P_{\eta}\right) \in \mathbb{Q}$ for all $\beta$, a claim which would be false at archimedean places, but it is evident from the proof that this is indeed the case.

Note that, as stated, the theorem applies when $f$ and $P$ are defined over the number field $K$, but the techniques are entirely local. If one has $f$ and $P$ defined over $\mathbb{C}_{v}$ then the same estimate holds, although of course only for the place $v$.

The proof of this comes in a sequence of lemmas. Roughly speaking, Lemma 7 establishes an inequality of the form (9) in a neighbourhood of each point in the support of the divisor $D=D(f, P)$ defined in (8). Since $\lambda_{X, D, v}$ will be bounded away from this support, it then suffices to show that $G_{f_{t}, v}\left(P_{t}\right)$ is as well (in the sense of $M_{K}$-boundedness). Lemma 9 establishes this in neighbourhoods of any points not in the support of $D$, but at which some coordinate of $P$ or some coefficient of $f$ has a pole. Once a neighbourhood of every such point has been removed, it is easy to show that $G_{f_{t}, v}\left(P_{t}\right)$ is appropriately bounded on the remainder of $X\left(\mathbb{C}_{v}\right)$, and this is the content of Lemma 8 .

Proof of Theorem 4. We will start by noting that we may replace $P$ by $f(P)$ if convenient, since we have

$$
G_{f_{t}, v}\left(f_{t}\left(P_{t}\right)\right)=d G_{f_{t}, v}\left(P_{t}\right)
$$

and

$$
D(f, f(P))=d D(f, P),
$$

whence

$$
\lambda_{X, D(f, f(P)), v}=d \lambda_{X, D(f, P), v} .
$$

In other words, proving Theorem 4 for $f(P)$ instead of for $P$ only decreases the implied constant. Without loss of generality, then, we may assume that for any $\beta \in X$ we have either $G_{f_{\eta}, \beta}\left(P_{\eta}\right)=0$ or $\log \left\|P_{\eta}\right\|_{\beta}>\mathcal{B}_{\beta}\left(f_{\eta}\right)$, from which Lemma 3 gives $G_{f_{\eta}, \beta}\left(P_{\eta}\right)=\log \left\|P_{\eta}\right\|_{\beta}$. We will also assume, since the quantities defined are independent of the choice of homogeneous coordinates, that $P_{N}=1$, and that $D$ is supported entirely on $K$-rational points (this last step amounting just to a finite extension of the ground field).

Note that it suffices to prove the estimate (9) on a arbitrary affine open $V \subseteq U$, since $X$ is quasi-compact, so we will assume that $D$ is given locally on $U$ by $g_{U}=0$, where $g_{U} \in \mathcal{O}(U)$ is regular. 
For each $\beta \in X(K)$ we will also fix a function $w_{\beta}$ with no poles other than at $\beta$. Given this, we will define

$$
\mathcal{D}_{v}(\beta ; r, R)=\left\{t \in X\left(\mathbb{C}_{v}\right): r<\frac{1}{\operatorname{deg}\left(w_{\beta}\right)} \log \left|w_{\beta}(t)^{-1}\right|_{v}<R\right\},
$$

the open annulus at $\beta$ of inner logarithmic radius $r$ and outer logarithmic radius $R$. For brevity we will set $\mathcal{D}_{v}(\beta ; r)=\mathcal{D}_{v}(\beta ;-\infty, r)$, and we note (to forestall possible confusion) that the radii are logarithmic.

Lemma 5. For any $\varphi \in K(X)$ there is an $M_{K}$-constant $\gamma$ such that

$$
\log ^{+}|\varphi(t)|_{v}=\frac{\log ^{+}|\varphi|_{\beta}}{\operatorname{deg}\left(w_{\beta}\right)} \log ^{+}\left|w_{\beta}(t)\right|_{v}+O_{v}(1)
$$

for all $t \in \mathcal{D}_{v}\left(\beta ; \gamma_{v}\right)$.

Proof. First we note that if $\varphi$ has no pole at $\beta$, then this reduces to the standard fact that $\varphi$ is bounded in a $v$-adic neighbourhood. We will assume that $\varphi$ has a pole, and hence that $\log ^{+}|\varphi|_{\beta}$ is the order of this pole.

Now,

$$
\log \left|\frac{\varphi^{\operatorname{deg}\left(w_{\beta}\right)}}{w_{\beta}^{\log ^{+}|\varphi|_{\beta}}}\right|=O_{v}(1)
$$

on $\mathcal{D}_{v}\left(\beta ; C_{v}\right)$ for some $M_{K}$-constant $C$, since the function on the left is regular at $\beta$. Unpacking, this becomes

$$
\log |\varphi(t)|_{v}=\frac{\log ^{+}|\varphi|_{\beta}}{\operatorname{deg}\left(w_{\beta}\right)} \log \left|w_{\beta}(t)\right|_{v}+O_{v}(1) .
$$

Since the implied constant vanishes for all but finitely many places, we may choose an $M_{K}$-constant $\gamma \leq C$ so that the right-hand-side is non-negative for all $t \in$ $\mathcal{D}_{v}\left(\beta ; \gamma_{v}\right)$, eliminating the distinction between (11) and (10).

Lemma 6. For any $\beta \in X$, there is an $M_{K}$-constant $\gamma$ such that

$$
\lambda_{X, D, v}(t)=\frac{\operatorname{ord}_{\beta}(D)}{\operatorname{deg}\left(w_{\beta}\right)} \log ^{+}\left|w_{\beta}(t)\right|_{v}+O_{v}(1)
$$

on $\mathcal{D}_{v}\left(\beta ; \gamma_{v}\right)$.

Proof. Choose an affine open $V$ containing $\beta$ on which $D$ is locally the divisor of $g_{V} \in K(X)$, and note that $-\log \left|g_{V}(t)\right|_{v}-\lambda_{X, D, v}(t)$ is locally bounded and continuous by definition. By Lemma 5, there is a $C_{V}$ such that

$$
-\log \left|g_{V}(t)\right|_{v}=\frac{\log ^{+}\left|g_{V}^{-1}\right|_{\beta}}{\operatorname{deg}\left(w_{\beta}\right)} \log \left|w_{\beta}(t)\right|_{v}+O_{v}(1)
$$

on $\mathcal{D}_{v}\left(\beta ; C_{V, v}\right)$. The estimate follows from this, since $\operatorname{ord}_{\beta}(D)=\log ^{+}\left|g_{V}^{-1}\right|_{\beta}$.

We may now take $\gamma$ to be the minimum of the $C_{V, v}$ as $V$ varies over the affine opens in some finite cover of $U$.

Lemma 7. Suppose that $\beta \in X$, and that $\log \left\|P_{\eta}\right\|_{\beta}>\mathcal{B}_{\beta}\left(f_{\eta}\right)$. Then there is an $M_{K}$-constant $\gamma$ such that for $t \in \mathcal{D}_{v}\left(\beta ; \gamma_{v}\right)$,

$$
G_{f_{t}, v}\left(P_{t}\right)=\frac{G_{f_{\eta}, \beta}\left(P_{\eta}\right)}{\operatorname{deg}\left(w_{\beta}\right)} \log ^{+}\left|w_{\beta}(t)\right|_{v}+O_{v}(1)
$$


Proof. By hypothesis there is some $i$ such that for every coefficient $a_{j, I}$ of $f$, the function $P_{i}$ has a pole at $\beta$ of order greater than that of $a_{j, I}^{1 / I_{N}}$, and that $G_{f_{\eta}, \beta}\left(P_{\eta}\right)=$ $\log \left|P_{i}\right|_{\beta}$. In other words, the function $P_{i}^{I_{N}} / a_{j, I} \in F$ has a pole of positive order at $\beta \in X$. By Lemma [5 we may choose an $M_{K}$-constant $C_{1, j, I}$ such that for some $M_{K}$-constant $C_{2, j, I}$ we have

$$
\log \left|P_{i}(t)\right|_{v} \geq \frac{1}{I_{N}} \log \left|a_{j, I}(t)\right|_{v}+\frac{1}{I_{N} \operatorname{deg}\left(w_{\beta}\right)} \log \left|\frac{P_{i}^{I_{N}}}{a_{j, I}}\right|_{\beta} \log ^{+}\left|w_{\beta}(t)\right|-C_{2, j, I, v}
$$

for all $t \in \mathcal{D}_{v}\left(\beta ; C_{1, j, I, v}\right)$. In particular, if $C_{3}$ is the $M_{K}$-constant $\gamma$ associated to $f$ by Lemma 3, then we can set

$$
C_{4, j, I}=\min \left\{C_{1, j, I},\left(C_{2, j, I}+C_{3}\right) \frac{I_{N}}{\log \left|P_{i} / a_{j, I}\right|_{\beta}}\right\}
$$

to ensure that

$$
\log \left|P_{i}(t)\right|_{v} \geq \frac{1}{I_{N}} \log \left|a_{j, I}(t)\right|_{v}+C_{3, v}
$$

for all $t \in \mathcal{D}_{v}\left(\beta ; C_{4, j, I, v}\right)$. Now if $C_{5}=\max _{j, I}\left\{C_{4, j, I}\right\}$ we have

$$
\log \left\|P_{t}\right\|_{v}>\mathcal{B}_{v}\left(f_{t}\right)+C_{3, v}
$$

for all $t \in \mathcal{D}_{v}\left(\beta ; C_{5, v}\right)$. Also note that, taking $\gamma \geq C_{5}$ large enough, we have $\left\|P_{t}\right\|_{v}=\left|P_{i}(t)\right|_{v}$ for all $t \in \mathcal{D}_{v}\left(\beta ; \gamma_{v}\right)$. By Lemma 3, for these values of $t$ we see

$$
\begin{aligned}
G_{f_{t}, v}\left(P_{t}\right) & =\log \left\|P_{t}\right\|_{v}+O_{v}(1) \\
& =\log \left|P_{i}(t)\right|_{v}+O_{v}(1) \\
& =\frac{\log \left|P_{i}\right|_{\beta}}{\operatorname{deg}\left(w_{\beta}\right)} \log ^{+}\left|w_{\beta}(t)\right|_{v}+O_{v}(1) \\
& =\frac{G_{f_{\eta}, \beta}\left(P_{\eta}\right)}{\operatorname{deg}\left(w_{\beta}\right)} \log ^{+}\left|w_{\beta}(t)\right|_{v}+O_{v}(1) .
\end{aligned}
$$

The following lemma is a standard application of the triangle inequality.

Lemma 8. For each $v \in M_{K}$, let $Z_{v} \subseteq U\left(\mathbb{C}_{v}\right)$, and suppose that the coefficients of $f$ and the coordinates of $P$ are all $M_{K}$-bounded on the $Z_{v}$. Then the functions $t \mapsto G_{f_{t}, v}\left(P_{t}\right)$ are $M_{K}$-bounded on the $Z_{v}$.

Proof. Suppose that the coefficients $a_{i, I}$ of $f$ satisfy $\log \left|a_{i, I}(t)\right|_{v} \leq C_{1, v}$ for $t \in Z_{v}$, and let $C_{2, v}=\log \left(\begin{array}{c}n+d-1 \\ d-1\end{array}\right)$ if $v$ is archimedean, and $C_{2, v}=0$ otherwise. Then for any $t \in Z_{v}$, the triangle inequality gives

$$
\log \left\|f_{t}\left(P_{t}\right)\right\|_{v} \leq \log \max \left\{\left|a_{i, I}(t) P_{t}^{I}\right|_{v}\right\}+C_{2, v} \leq d \log \left\|P_{t}\right\|_{v}+C_{1, v}+C_{2, v} .
$$

By induction we have

$$
d^{-n} \log \left\|f_{t}^{n}\left(P_{t}\right)\right\|_{v} \leq \log \left\|P_{t}\right\|_{v}+\frac{1}{d-1}\left(C_{1, v}+C_{2, v}\right) .
$$

The lemma follows by taking $n \rightarrow \infty$, and noting that the coordinates of $P$ are (by hypothesis) $M_{K}$-bounded on the $Z_{v}$.

Lemma 9. Suppose that $\beta \in X(\bar{K})$ and that $G_{f, \beta}(P)=0$. Then there in an $M_{K}$-constant $\gamma$ such that we have $G_{f_{t}, v}\left(P_{t}\right)=O_{v}(1)$ for $t \in \mathcal{D}_{v}\left(\beta ; \gamma_{v}\right)$. 
Proof. First we establish a bound for any given place $v \in M_{K}$, and later we will show that this bound is almost always zero (and hence the local bounds piece together to an $M_{K}$-constant bound).

We will choose a uniformizer $\pi \in F$ at $\beta$, and note that any function $\varphi \in F$ admits a Laurent series expansion

$$
\varphi=a_{m} \pi^{m}+a_{m+1} \pi^{m+1}+\cdots,
$$

where $a_{i} \in \bar{K}, m \in \mathbb{Z}$, and $a_{m} \neq 0$ (from which $m=-\log |\varphi|_{\beta}$ ). Now, choose $0<\varepsilon<1$ so that the series for the coordinates of $P_{i}$ and the coefficients of $f$ all converge on $0<|\pi|_{v}<\varepsilon^{1 / 2}$. Since $\varepsilon<\varepsilon^{1 / 2}$, these functions all have a maximum $v$-adic absolute value on

$$
S_{\varepsilon}=\left\{z \in X\left(\mathbb{C}_{v}\right):|\pi(z)|_{v}=\varepsilon\right\} .
$$

Let $P_{i, n}$ denote the $i$ th coordinate of $f^{n}(P)$. In particular, by (12) there is a constant $B_{v} \geq 0$ such that $\log \left|P_{i, n}(t)\right|_{v} \leq d^{n} B_{v}$ on $S_{\varepsilon}$. Also,

$$
\pi^{\log ^{+}\left|P_{i, n}\right|_{\beta}} P_{i, n} \in F
$$

is regular on $|\pi|_{v} \leq \varepsilon$, and so attains its maximum value on $S_{\varepsilon}$. It follows, if

$$
A_{\delta, \varepsilon}=\left\{z \in X\left(\mathbb{C}_{v}\right): \delta \leq|\pi(z)|_{v} \leq \varepsilon\right\},
$$

for any given $0<\delta<\varepsilon$, that

$$
\delta^{\log ^{+}\left|P_{i, n}\right|_{\beta}}\left|P_{i, n}(t)\right|_{v} \leq\left|\pi(t)^{\log ^{+}\left|P_{i, n}\right|_{\beta}} P_{i, n}(t)\right|_{v} \leq \varepsilon^{\log ^{+}\left|P_{i, n}\right|_{\beta}} d^{n} B_{v},
$$

for $t \in A_{\delta, \varepsilon}$, whence

$$
\log \left|P_{i, n}(t)\right|_{v} \leq \log ^{+}\left|P_{i, n}\right|_{\beta} \log (\varepsilon / \delta)+d^{n} B_{v} .
$$

Since this is true for all $i$, we have

$$
G_{f_{t}, v}\left(P_{t}\right)=\lim _{n \rightarrow \infty} d^{-n} \log \left\|f_{t}^{n}\left(P_{t}\right)\right\|_{v} \leq \lim _{n \rightarrow \infty} d^{-n} \log \left\|f_{\eta}^{n}\left(P_{\eta}\right)\right\|_{\beta} \log (\varepsilon / \delta)+B_{v}=B_{v}
$$

for $t \in A_{\delta, \varepsilon}$, since $G_{f_{\eta}, \beta}\left(P_{\eta}\right)=0$. But this bound is independent of $\delta$, and hence holds on

$$
\bigcup_{0<\delta<\varepsilon} A_{\delta, \varepsilon}=S_{\varepsilon} \backslash\{\pi=0\} .
$$

By Lemma 5 there is a $\gamma_{v} \in \mathbb{R}$ such that $\mathcal{D}_{v}\left(\beta ; \gamma_{v}\right) \subseteq S_{\varepsilon} \backslash\{\pi=0\}$. In other words, we have $G_{f_{t}, v}\left(P_{t}\right)$ bounded on some set of the form $\mathcal{D}_{v}\left(\beta ; \gamma_{v}\right)$.

It remains to show that for all but finitely many $v \in M_{K}$, we may take $B_{v}=0$ in the above argument, and $\gamma_{v}=0$.

Fix a function $\varphi$ on $X$. If $v$ is a non-archimedean place at which the coefficients of the series defining $\varphi$ are integral, and the leading coefficient is a unit, then this series converges on $0<|\pi(t)|_{v}<1$. Note that this is a condition met for all but finitely many places, by Lemma 7 of [6]. So, given that $v$ is a place of this description, we have

$$
|\varphi(t)|_{v}=|\pi(t)|_{v}^{-\log |\varphi|_{\beta}}
$$

for all $t$ with $0<|\pi(t)|_{v}<1$. So, applying this to each coefficient of $f$ and each coordinate of $P$, and assuming that the series for each meets the conditions of $v$ integrality, we see that every one of these functions $\varphi$ satisfies $|\varphi(t)|_{v}=\varepsilon^{-\log |\varphi|_{\beta}}$ on $S_{\varepsilon}$, for any $0<\varepsilon<1$, and so in particular we may take $B_{v}=-\kappa \log \varepsilon$ for some constant $\kappa$ which is independent of $\varepsilon$. This now gives the bound

$$
G_{f_{t}, v}\left(P_{t}\right) \leq-\kappa \log \varepsilon
$$


for $t \in A_{\delta, \varepsilon}$, for any $0<\delta<\varepsilon<1$. As above, since this bound is independent of $\delta$, it holds on $A_{0, \varepsilon}$. But note that, for $\varepsilon \leq \varepsilon^{\prime}<1$, we have $A_{0, \varepsilon} \subseteq A_{0, \varepsilon^{\prime}}$, and so we have $G_{f_{t}, v}\left(P_{t}\right) \leq-\kappa \log \varepsilon^{\prime}$ on $A_{0, \varepsilon}$. This holds for an arbitrary $\varepsilon \leq \varepsilon^{\prime}<1$, and so taking $\varepsilon^{\prime} \rightarrow 1$, we have $G_{f_{t}, v}\left(P_{t}\right)=0$ on $A_{0, \varepsilon}$. This is now independent of $\varepsilon$, and so we have the same on $A_{0,1}$.

So, for all but a finite set of places $v$, we have $G_{f_{t}, v}\left(P_{t}\right)=0$ on $0<|\pi(t)|_{v}<$ 1. But by Lemma [5, for all but finitely many $v$ the latter set coincides with $\mathcal{D}_{v}(\beta ; 0)$.

We are now in a position to finish the proof of Theorem 4. Let $S \subseteq X$ be the set of points at which any coordinate of $P$ or any coefficient of $f$ has a pole, and let $U=X \backslash S$. Note that $\operatorname{Supp}(D) \subseteq S$, from Lemma 3 if nothing else. For each $\beta \in \operatorname{Supp}(D)$, Lemmas 6 and 7 allow us to choose $M_{K}$-constants $\gamma_{\beta}$ and $C_{1, \beta}$ such that

$$
\left|G_{f_{t}, v}\left(P_{t}\right)-\lambda_{X, D, v}(t)\right| \leq C_{1, \beta, v}
$$

on $\mathcal{D}_{v}\left(\beta ; \gamma_{\beta, v}\right)$. We let $C_{1}=\max _{\beta \in \operatorname{Supp}(D)} C_{\beta}$.

Now, by standard arguments for local heights, we may choose an $M_{K^{-}}$-constant $C_{2}$ such that

$$
\lambda_{X, D, v}(t) \leq C_{2, v}
$$

whenever

$$
t \notin Z:=\bigcup_{\beta \in \operatorname{Supp}(D)} \mathcal{D}_{v}\left(\beta ; \gamma_{\beta, v}\right) .
$$

Specifically if $D$ is given locally on the affine patch $V \subseteq U$ by $g_{V}$ (regular since $D$ is effective), then Lemma 8 of [6] ensures the existence of an $M_{K}$-divisor $C_{V}$ such that $\left|g_{V}(t)\right|_{v}<C_{V, v}$ implies $\left|w_{\beta}(t)^{-1}\right|_{v}<\operatorname{deg}\left(w_{\beta}\right) \gamma_{\beta, v}$ for some $\beta \in \operatorname{Supp}(D)$. This bounds $\lambda_{X, D, v}(t)$ for $t \in V \backslash Z$, and combining the bounds for some finite affine cover of $X$ gives the claim. It suffices to similarly bound $G_{f_{t}, v}\left(P_{t}\right)$ on $U\left(\mathbb{C}_{v}\right) \backslash Z$.

Lemma 9 provides, for each $\beta \in S \backslash \operatorname{Supp}(D)$, constants $\gamma_{\beta}$ and $C_{3, \beta}$ such that

$$
G_{f_{t}, v}\left(P_{t}\right) \leq C_{3, \beta, v}
$$

for $t \in \mathcal{D}_{v}\left(\beta ; \gamma_{\beta, v}\right)$. But again by Lemma 8 of [6], the coordinates of $P$ and the coefficients of $f$ are $M_{K}$-bounded on the complement of $\bigcup_{\beta \in S} \mathcal{D}_{v}\left(\beta ; \gamma_{\beta, v}\right)$, and so by Lemma 8 we have an $M_{K}$-constant $C_{4}$ such that

$$
G_{f_{t}, v}\left(P_{t}\right) \leq C_{4}
$$

on this set.

Now, take

$$
C=\max \left\{\max _{\beta \in \operatorname{Supp}(D)}\left\{C_{1, \beta}\right\}, \max _{\beta \in S \backslash \operatorname{Supp}(D)}\left\{C_{2}+C_{3, \beta}\right\}, C_{2}+C_{4}\right\},
$$

an $M_{K}$-constant. By (13), (14), (15), and (16) we have

$$
\left|G_{f_{t}, v}\left(P_{t}\right)-\lambda_{X, D, v}(t)\right| \leq C_{v}
$$

for all $t \in U$. 


\section{PRoOf OF THE MAIN RESUlt}

Before proceeding with the bulk of the proof of Theorem 1, we note that there is no injunction against the image of $P: X \rightarrow \mathbb{P}^{N}$ landing entirely in the invariant hyperplane, in which case the Green's functions developed in Section 1 will be entirely useless. Fortunately, in this special case Theorem 1 reduces to well-known facts about heights.

Lemma 10. Let $f: \mathbb{P}^{N} \rightarrow \mathbb{P}^{N}$ be a morphism defined over $K$, and let $P: X \rightarrow \mathbb{P}^{N}$ be a morphism. Then

$$
\hat{h}_{f}\left(P_{t}\right)=\hat{h}_{f}\left(P_{\eta}\right) h_{X, D}(t)+O(1),
$$

for some divisor $D \in \operatorname{Div}(X) \otimes \mathbb{Q}$ of degree 1 .

Proof. Using the basic properties of the canonical height [3, and the functoriality of heights with respect to morphisms [5, p. 184], we have

$$
\begin{aligned}
\hat{h}_{f}\left(P_{t}\right) & =h_{\mathbb{P}^{N}, H}\left(P_{t}\right)+O_{f}(1) \\
& =h_{X, P^{*} H}(t)+O_{f, P}(1) .
\end{aligned}
$$

It remains to show that $\operatorname{deg}\left(P^{*} H\right)=\hat{h}_{f}\left(P_{\eta}\right)$ in this special case. But this is straightforward in light of the results above. In particular, $\mathcal{B}_{v}(f)=0$ for any place $v$ of the function field $K(X)$, and hence $G_{f, v}(P)=\log ^{+}\left|P_{\eta}\right|_{v}$ for any place $v$. Summing over all places, we see that $\hat{h}_{f}\left(P_{\eta}\right)=h\left(P_{\eta}\right)=\operatorname{deg}(P)$.

Now, if the map $P: X \rightarrow \mathbb{P}^{N}$ lands entirely in the hypersurface $H \subseteq \mathbb{P}^{N}$, we may compute the canonical height by restricting the problem to $H$. Our hypothesis that the family is fibral ensures, after a change of variables, that it retricts to a constant family of maps on $H \cong \mathbb{P}^{N-1}$. Since the canonical height is invariant under change of coordinates, and since composing with this change of coordinates preserves the condition that the image of $P$ lies in $H$, Lemma 10 now gives us Theorem 1 . So we may assume from here on out that the image of $P$ is not contained in $H$, and so there are in fact only finitely many points $t \in X$ such that $P_{t} \in H$.

Given this restriction, we now define

$$
D(f, P)=\sum_{\beta \in X} G_{f_{\eta}, \beta}\left(P_{\eta}\right)[\beta] \in \operatorname{Div}(X) \otimes \mathbb{Q}
$$

as above. We note that, by the basic properties of the Green's functions established in Section 1, $D(f, P)$ is an effective divisor of degree $\hat{h}_{f_{\eta}}\left(P_{\eta}\right)$. Theorem 1 follows from summing the estimate in Theorem 4 over all places of $K$, or any finite extension thereof, and by taking the divisor in the statement to be $D(f, P) / \operatorname{deg}(D(f, P))$.

Corollary 2 follows from the fact [7, Proposition 5.4, p. 115] that, for any two divisors $D, E$ of the same degree on $X$, we have

$$
h_{X, D}(t)=h_{X, E}(t)+O\left(h_{E, X}(t)^{1 / 2}\right),
$$

with an improvement in the error to $O(1)$ when $X$ is rational.

\section{REFERENCES}

[1] M. Baker and L. DeMarco, Preperiodic points and unlikely intersections. Duke Math. J. 159 (2011), no. 1, pp. 1-29.

[2] E. Bedford and M. Jonsson, Dynamics of regular polynomial endomorphisms of $\mathbf{C}^{k}, A m e r . J$. Math. 122 (2000), pp. 153-212. 
[3] G. S. Call and J. H. Silverman, Canonical heights on varieties with morphisms, Compositio Math. 89 (1993), pp. 163-205.

[4] D. Ghioca and N. M. Mavraki, Variation of the canonical height in a family of rational maps, New York Journal of Mathematics 19 (2013), pp. 873-907.

[5] M. Hindry and J. H. Silverman, Diophantine Geometry: An Introduction, volume 201 of Graduate Texts in Mathematics, Springer, 2000.

[6] P. Ingram, Variation of the canonical height for a family of polynomials, J. Riene Ange. Math. (to appear).

[7] S. Lang, Fundamentals in Diophantine Geometry, Springer, New York, 1983.

[8] J. Tate, Variation of the canonical height of a point depending on a parameter. Amer. J. Math. 105 (1983), no. 1, pp. 287-294.

Colorado State University, Fort Collins, Colorado, USA

E-mail address: pingram@math.colostate.edu 